\section{Research}

\section{"Corresponding author}

Miguel Sebastian Egoavil, MD

Unit of Biomedical Informatics in

Global Health

Universidad Peruana Cayetano Heredia

Av. Honorio Delgado 430 - Lima 31

15102, Lima, Perú

Tel. +5114818283

E-mail: miguel.egoavil@upch.pe

Volume 3 : Issue 2

Article Ref. \#: 1000PMHCOJ3122

\section{Article History}

Received: March 1 $1^{\text {st }}, 2017$

Accepted: June 29 $9^{\text {th }}, 2017$

Published: June $29^{\text {th }}, 2017$

\section{Citation}

Egoavil MS, Condor DF, PinazoVidal MA, et al. Qualitative study for the development of a telemedicine system in palliative care. Palliat Med Hosp Care Open J. 2017; 3(2): 14-21. doi: 10.17140/PMHCOJ-3-122

\title{
Qualitative Study for the Development of a Telemedicine System in Palliative Care
}

Miguel Sebastian Egoavil, MD ${ }^{1,4^{*}}$; Daniel Flavio Condor, BSc ${ }^{1,4}$; Miguel Alonso PinazoVidal, MD'; Juan Manuel Quezada, BSc ${ }^{1}$; Boris Manuel Fazio, BSc ${ }^{2}$; Juan Carlos Bueno, MS $^{3}$; Luis Enrique Peña, MS ${ }^{3}$; Jaime Farfán, MS $^{3}$; Jose Gaspar De la Puente, MS $^{3}$; Cesar Arce, $\mathrm{Mr}^{3}$; Bregy Malpartida, $\mathrm{Mr}^{3}$; Anthony Arostegui, $\mathrm{Mr}^{3}$; Jose Enrique Pérez-Lu, $\mathrm{PhD}^{2}$

${ }^{1}$ Biomedical Informatics in Global Health Unit, Facultad de Salud Pública y Administración, Universidad Peruana Cayetano Heredia, Lima, Perú

${ }^{2}$ Ministry of Health, Lima, Perú

${ }^{3}$ Departamento de Ingeniería Industrial, Universidad de Ingeniería y Tecnología, Lima, Perú ${ }^{4}$ Epidemiology, HIV and STI Unit, Facultad de Salud Pública y Administración, Universidad Peruana Cayetano Heredia, Lima, Perú

\section{ABSTRACT}

Introduction: Technological advances in public health have allowed the life expectancy of people to increase. However, new challenges appear in populations that age as the greatest number of chronic and oncological diseases requiring chronic and/or palliative care. In Peru, this is an area that has not developed. The use of Information and Communication Technologies (ICT) can optimize healthcare, and in the case of palliative care they could shorten the distances between the specialist, the caregiver and the patient.

Objective: To know the needs of health professionals and caregivers of patients who are in palliative care, regarding the management of information, knowledge, attitudes and the use of ICT in palliative care.

Materials and Methods: As part of the TeleJampiq project, a first phase of qualitative study is developed. A focus group was conducted with six caregivers. Family members' information was obtained through two field reports and two interviews. Finally, the exploration of the needs and suggestions of palliative care specialists was carried out by ten in-depth interviews (six physicians, one psychologist and three nurses). Through the data analysis we were able to identify three major themes in which the results were classified: Management of information on chronic diseases and terminal patients, knowledge and actions in palliative care and use of ICT for palliative care.

Results: Management of information on chronic diseases and terminal patients. It is essential to draw a distinction between terminability and palliation. Managing these concepts could make a difference from the start of treatment. Knowledge and actions in palliative care. Nonfamily caregivers' knowledge of the meaning of palliative care is like that of specialists (doctors and nurses). Use of ICT for palliative care. The use of mobile devices among health personnel interviewed is overwhelming. Engaging in aspects of information management to other specialists such as nutritionists and psychologists would be beneficial.

Conclusions: It is necessary to use ICT in the field of chronic and palliative care, being a fundamental aspect the communication between personnel working in the system and the use of scales, care protocols, among others.

KEY WORDS: Telemedicine; Interviews as topic; Focus groups; Palliative care.

\section{Copyright}

(C2017 Egoavil MS. This is an open access article distributed under the Creative Commons Attribution 4.0 International License (CC BY 4.0), which permits unrestricted use, distribution, and reproduction in any medium, provided the original work is properly cited.
ABBREVIATIONS: ICT: Information and Communication Technologies; ADAMO: Medical Support Service Home care of the elderly and oncological patient; Oncosalud: Private clinic center specialized in cancer and palliative care; EHR: Electronic Health Records. 


\section{INTRODUCTION}

In the last millennium, people's life expectancy has essentially doubled due to improvements in living conditions, technological advances and public health measures. ${ }^{1,2}$ This has enabled control of infant mortality and deaths from infectious diseases globally. However, coincidentally in this last millennium we have also seen the emergence of a new epidemic: chronic diseases and cancer. These diseases have been favored by aspects such as rapid urbanization, environmental pollution, unhealthy lifestyles and the aging of populations. These chronic diseases and cancer often known as non-communicable diseases (NCDs) as a whole - affect at least 36 million people worldwide, causing disability, chronic problems, prolonged periods of agony and finally death. ${ }^{3}$ In the case of patients with advanced disease, with no possibility of cure or improvement, the management offered is called "palliative care" and focuses on improving the quality of life (QoL) of the patient and those around him. Palliative care involves the prevention and relief of suffering, such as the treatment of pain, optimizing physical, psychosocial, and depending on spiritual situations. ${ }^{4,5}$

In high income countries, there is a great knowledgeabout palliative care, which was initially carried out in an institutionalized way, either through general hospitals or specially structured hospitals (hospices). ${ }^{2}$ Progressively, these services have been decentralized and created specialized teams and services that support home management, which can be more comfortable, efficient and humane. ${ }^{6,7}$ In countries with similar economic status such as ours, medium- and low-income countries, still overwhelmed by unresolved public health problems such as infectious diseases, with scarcity and poor distribution of human resources and health services infrastructure, the response to chronic diseases and cancer is still incipient. ${ }^{4}$ This causes patients not to receive an adequate treatment or that their pain, discomfort or complications cannot be efficiently managed. In addition, this increases the number of visits to health facilities due to the continuous decompensation, saturating emergency departments and increasing the number of days these patients have to be hospitalized. This problem not only occurs in Peru, but is a problem that concerns other countries in Latin America and even developed countries such as Germany. ${ }^{8}$

Information and communication technologies (ICT) in their various manifestations and their use in healthcare can benefit chronic patients and improve the supply of palliative care services. ${ }^{9,10}$ For example, electronic health records (EHR) can store higher quality data, ${ }^{11}$ have good acceptability by users, ${ }^{12,13}$ improve healthcare, ${ }^{10}$ and can send alerts and reminders for support of medical decisions. ${ }^{14}$ In terms of telemedicine, which is the provision of distance medical services to improve the health of patients through the use of a device that involves the use of ICT, ${ }^{15}$ it can have a significant impact on the improvement of the quality of patient care, improved coverage, and improved efficiency in terms of cost and performance compared to the traditional health system, especially when it refers to specialized services. ${ }^{6,16}$
At the present time, telemedicine applied to the palliative care of patients is a poorly explored field, ${ }^{6,17}$ but there is evidence suggesting that it could improve the quality of patient care, communication with health personnel, a decrease of the documentation used to collect information and reduce costs. But there is still a lot to develop and explore, including studies with qualitative designs that allow the evaluation of the different perspectives of the actors involved in palliative care and their opinion about the use of ICTs in this type of services. ${ }^{18}$ For this reason, and as part of the first phase of the TeleJampiq project, which is a "Telemedicine System for patient monitoring and decision-making in palliative care", we conducted the present qualitative study with the objective of knowing the needs of physicians, caregivers and relatives of patients in palliative care and their perspective on the use of telemedicine and ICTs in care.

\section{METHODS}

The data collection was carried out from three sources: nonfamily caregivers (non-professional health personnel), family caregivers and palliative care specialists. Participants belong to the medical support service home care of the elderly and oncological patient (ADAMO) that is supported by the Callao Region Government and the Oncosalud clinic which is a private clinic in Lima, specialized in cancer prevention, diagnosis and treatment. These elected institutions represent the two main scenarios of palliative care in our country. On the one hand we have ADAMO, a public entity that provides services to patients of low and medium resources; and on the other hand, Oncosalud, an entity whose assigned population is in the capacity to pay private health insurance.

With regard to the research method chosen, focus groups of 6 non-family caregivers and 4 family caregivers were conducted. The information of family caregivers was also obtained through field reports and interviews in visits to patients and relatives. Finally, the exploration of the needs and suggestions of the specialists in the subject was carried out in 10 detailed in-depth interviews (6 doctors, 1 psychologist and 3 nurses).

Prior verbal informed consent was given to the participants, who could remain or leave the study group if they did not have their consent. The study protocol was approved by the institutional review board (IRB) of the Universidad Peruana Cayetano Heredia and the Callao Region Health Department (Table 1).

The objective of the focus groups was to know the perceptions of non-family caregivers and family caregivers about chronic and oncological diseases, patients in the terminal phase or those who cannot stand for themselves and the care they receive, as well as knowing their needs and suggestions for improved monitoring in these patients. The topics discussed and questions asked during the focus groups are specified in Table 2.

The interview guide to health staff sought to gain in- 
sight into the care of patients in palliative care, In addition, to know the needs and suggestions for the improvement of monitoring, as well as the perception towards the use of the technological systems in the follow-up of these patients. This guide was composed in two topics as described in Tables 3 and 4.
The information was used to make a comparative analysis of the testimonies and data obtained from the groups and it was divided into three themes identified: Management of information on chronic and oncological diseases, knowledge and actions in palliative care and the use of information and com-

\begin{tabular}{|cccc}
\hline \multicolumn{2}{|l}{ Table 1: Data Sources of Information. } & \\
\hline Source & Type of qualitative approach & Institution & $\mathbf{n = 2 0}$ \\
\hline Non-family caregivers & Focus Group & Oncosalud & 6 \\
\hline Family caregivers & Focus Group - Interviews & Adamo & 4 \\
\hline \multirow{2}{*}{ Specialists } & In-depth Interviews & Oncosalud & 6 \\
\cline { 2 - 4 } & & Adamo & 4 \\
\hline
\end{tabular}

Table 2: Topics and Questions for the Focus Groups.

Family Caregivers Non-family Caregivers

Topic 1: Chronic diseases, oncological diseases, and terminally ill patients

1. We would like to know what you think about chronic diseases. What does it mean to you?

2. In the same way, we would like to know what do you think or understand about cancer-related diseases. What does it mean to you?

3. We know that there are a variety of chronic and oncological diseases. We want to find out which ones you consider to be more serious. Why do you think so?

4. We know that the adult population is susceptible to several diseases. What do you think about it?

5. Once we understand that both are types of diseases, we would like to know what you understand by terminally ill patients. What does it mean to you?

6. What care do you give to terminally ill patients?

7. How do you think it affects the family to have a patient with these diseases?

Topic 2: Palliative/chronic care

1. What do you understand about palliative or chronic care?

2. We know that terminally ill patients need palliative care. What is the most important thing for you when caring for a patient in palliative care?

3. What activity do you frequently do when caring for a patient in palliative care?

4. What do you understand by patient monitoring?

5. What kind of help would you require from health professionals to improve care for these patients?

6. What aspects should be taken into account as to the conditions that should be at home to have a patient with these diseases?

6. How often do these patients visit the emergency room?

7. What kind of information should family members of patients in palliative care receive?

Topic 3: Perceptions on the use of ICT in palliative care

1. How many of you have used a tablet or a smartphone? What do you use these devices for?

2. Do you know what we mean when we talk about apps in this type of devices? What apps do you use frequently?

3. What do you think about apps for health care?

4. What do you think of using these apps for patient monitoring in palliative care?

5. Apart from having a device for patient monitoring, what other needs would you have?

6. What suggestions do you have for the development of a system for patient monitoring in palliative care?

7. What patient information is important?

8. What information do you frequently need?

9. What kind of alarms would you like to receive? What information?

10. How would you like to monitor your family member's pain?

11. How would you like to review your relative's information?

10. How would you like to monitor the patient's pain?

11. How would you like to review your patient's information?

12. Who else should see the information obtained from the patient?

13. In what palliative care topics should caregivers be trained? What topics do you suggest?

13. What do you think of being trained through video tutorials?

14. What palliative care topics would you like to be trained in? What topics do you suggest? 


\begin{tabular}{l}
\hline Table 3: First Topic for the Deep-Interviews. \\
\hline Perceptions related to palliative care \\
\hline 1. What do you understand about palliative care? \\
\hline 2. What is the most important when caring for a patient in palliative care? \\
\hline 3. What activities do you recommend doing frequently when caring for a patient in palliative care? \\
\hline 4. What do you mean by patient monitoring? \\
\hline 5. What should be monitored in the patient in palliative care? \\
\hline 6. How often do patients visit the emergency due to complications in their state of health? \\
\hline
\end{tabular}

\begin{tabular}{rl|}
\hline Table 4: & Second Topic for the Deep-Interviews. \\
\hline 1. & Have you ever used a tablet or a smartphone? How often do you use this type of device? \\
\hline 2. & What applications of these devices do you use frequently? \\
\hline 3. & What do you think about the applications for health care? \\
\hline 4. & What do you think of using these applications for patient monitoring in palliative care? \\
\hline 5. & Aside from having a device described above, what other needs would you have to use a system for patient monitoring in palliative care? \\
\hline 6. & What suggestions do you have for the development of a system for patient monitoring in palliative care? \\
\hline 7. & What patient data is so important that you need to know them immediately? \\
\hline 8. & What information do you frequently need? \\
\hline 9. & What kind of alarms would you like to receive, in what form and by what means? \\
\hline 10. & How would you like to monitor the patient's pain? \\
\hline 11. & How would you like to review patient information? \\
\hline 12. & Who else should see the information that is obtained from the patient? \\
\hline 13. & In what palliative care topics should caregivers be trained, whether they are family members or untrained health personnel? What topics do you suggest? \\
\hline 14. & Do you think technology can support adherence to treatment by the patient? \\
\hline
\end{tabular}

munication technologies for palliative care.

\section{RESULTS}

\section{Management of Information on Chronic and Oncological Diseases}

Most of the non-family caregivers (technical staff) who participated in the focus group had several years working in the field and handled pertinent information on chronic and oncological diseases based on their experience. They understand chronic diseases as those that persist in time. Per the experience gained in treating people in this situation, the most serious diseases are identified as head and neck neoplasms, since they are usually the most complicated because of their effects and the suffering that can cause the patient. Non-family caregivers agree that complications are related to the patient's advanced age and lack of family support.

Regarding the care of patients in the terminal phase, they call attention to the importance of providing them with quality of life (QoL). They also affirm the importance of pre- paring the patient's family to face the nearness of death. They specify that psychological support is important because complications arise due to the lack of family support ties or the feeling of loneliness and abandonment.

"Because we do not always work in these aspects, we simply go to the clinical, physical... The emotional and psychological part of these people is sometimes left aside... Make them understand these things, what is happening to his relative, they have to understand that it will happen, it has to happen, it is not eternal. And that helps to dispel their sorrows, to understand that, and our help is like that."

It is essential to draw a distinction between terminability and palliation. Since terminability is a sub-classification of palliative care:

"palliatives have several classifications. The terminal patient is focused only in the last 6 months of life. That is why the approach of a palliative is not the same as a terminal. A palliative can be longer than a terminal if I really want to cure it" (Total 
Care Doctor).

The emerging theme identified in this section came from the focus group, where some references to non-systematic types of knowledge arose from experience in the care of terminally ill patients. Non-family caregivers claim to recognize the agonizing or "sopor" phase by the skin characteristics and odor in patients. This knowledge allows them to anticipate to the moment of death and prepare the family.

\section{Knowledge and Actions in Palliative Care}

The non-family-caregivers' knowledge of what palliative care means is like that of the specialists (doctors and nurses); however, it may be noted that while caregivers refer to this knowledge based on their experience, since they cite specific cases, specialists turn to more theoretical concepts. However, both types of knowledge define the basic principles of palliative care. Some caregivers describe palliative care as:

"More than all, to provide QoL, to relieve all the symptoms that could present the patient, in this case, the main symptom in a cancer patient is the pain that we have to relieve, so that this patient is comfortable and, more than anything, relief from something that overwhelms them and give them all the best, that's what I think."

While experts indicate:

"Palliative care is to provide care to patients who are in the stage of disease progression. Generally, what we do is to alleviate, to diminish the symptoms that are in progression. Then, the idea is to improve the QoL, not only in the physical aspect but the whole environment. The emotional, spiritual and family environment."

The actions recommended by the specialists are oriented to: inform the relatives about the characteristics of the disease and the care required by the patient, especially the family caregiver; To maintain a horizontal and constant communication between relatives, technicians and specialists considering psychological and physical aspects; and to consider aspects such as: symptom management, pain scale score, prevention of ulcers and bedsores, hygiene, feeding, medication administration, alarm signs, among others.

The psychologist who participated in this phase indicates the importance of distracting activities to improve the predisposition of the family caregiver in the care of the patient to reduce the stresses of both, which are increasing as the disease progresses.

Both caregivers and specialists give emphasis to the importance of a correct patient monitoring, that is, the continuous monitoring of the symptoms presented, the review of vital functions, recording the scale of pain, among others; giving spe- cial care to the management of pain as a theme to highlight in the analysis of information.

They also indicate that the unnecessary monitoring which involving physical manipulation should not be performed in terminally ill patients because it impairs their comfort and may further deteriorate their health. This is related to the identification of stages in the evolution of the patient's disease: terminal, pre-agonist and agonistic or "sopor".

\section{Use of ICT for Palliative Care}

The use of mobile devices among health professionals interviewed is overwhelming; some refer to use simple phones while others use smartphones. In both cases the most frequent use is for personal matters, although they also use it for work matters. Both caregivers and medical specialists say that in some way they use their mobile devices to facilitate their work, either through telephone calls, to update certain data from the patient's home to the health institution or to confirm appointments.

"Then, with the family we have had contact by Skype by Whatsapp ${ }^{\mathrm{TM}}$ or they tell me 'I got a wound' and they send me the photo. And I ask them 'what do you have there?' Because it is not the same what they have there than what you have here" (Nurse from the "Total Care"program).

On the other hand, in the family environment of the patients and among the patients themselves, the use of mobile technologies is more limited, especially between families with more humble economic conditions. From the visits made to patients of the ADAMO program, it was also possible to verify that the elderly relatives usually reject the use of mobile devices or in some cases show no greater interest.

The perceptions about the use that can be given to these devices for the care of patients receiving palliative care, specifically for the monitoring of the patient, would depend on the stage of illness in which the patient is, for example, in the use of vital signs sensors the opinions were divided, for some interviewees these would not be adequate in palliative care patients in general, although others mention that they could serve only in the early stages and not in the pre-agonic phase, mainly because these devices can disturb the patient; also indicate that the recording of symptoms depends on the situation of each patient. The sensors that could be used in the indicated stages, mentioned by the majority, were a manometer, a heart rate and oximetry meter, patient position sensor, glucometer (exclusive for diabetic patients, as one interviewee points out), airflow sensor and thermometer. They also mentioned that it would be important to use pain scales for patient monitoring.

About the management of information, there is a consensus among health professionals and caregivers that it would be very helpful to have it digitized as an Electronic Health Record (EHR), both for recording all primary patient information 
and for recording symptoms, reporting pain scales, among others.

"With the experience we have we realize that it is very important to know almost everything about the patient. For example, when we go to a patient's house sometimes we do not have data, his last analyzes, and how much was his hemoglobin, especially to see the leukocytes because he has done chemotherapy. Suddenly the defenses are low or in neutropenia. All of this is important for us to have all the data, the whole history of the patient. So we can be able to educate the family... Another thing is that information has to be available for everyone. Because when you arrive to the patient's home, you store some information, but the next one (health professional) that goes have to know all that information. Because you cannot ask the same questions again because that generate insecurity in the patient and their relatives" (Nurse from Oncosalud).

One important theme that also arises from the in-depth interviews is the multidisciplinary dimension for palliative care. Engaging in other aspects of information management to other specialists, such as nutritionists and psychologists, would be beneficial as digitized access to specific patient information would help them in their work.

"It is important to me to see how the patient is evolving, whether or not my subject, because that would tell me more or less how the patient's mood will vary too, right? It gives me an idea of, perhaps what the patient might or might not need at that time, then, medical or nursing information does work for me, right? Yes, I would care to know what he does during the day. I do not know, for example, a record of whether get up from bed for breakfast or had breakfast in bed, and that for me is important, or if today was showered or not showered, those things for me as a psychologist are important" (Psychologist from ADAMO program).

Finally, the health staff agrees to point out that an indispensable element would be to have tutorials or guides to which both family members and technical caregivers can access for adequate management of symptoms and on some other aspects relevant to care, which also include components of psychological help. The use of technological devices can also, as experts say, serve for appointment reminders, drug administration and assess adherence.

\section{DISCUSSION}

The implementation of a telemedicine system for the monitoring and decision-making of the patient in chronic and palliative care is a complex and delicate process and as such, should start by addressing the needs of all those involved in healthcare. In this study, we conducted interviews, field visits and focus groups with palliative care physicians, general practitioners and caregivers of oncology patients. The participants identified the knowledge they have acquired throughout their professional practice and the attitudes or actions they take with respect to the palliative care of their patients. The findings are presented in three different themes: Management of information on chronic and oncological diseases, knowledge and actions in palliative care and the use of information and communication technologies for palliative care.

The adequate management of information on palliative care is determinant for the patient's well-being at home. ${ }^{19}$ As participants mentioned, knowledge is important because it is the basis of the design of tools for the monitoring of patients, to provide QoL. Also, it allows anticipating to the different complications that can appear in the care of this type of patients. On the other hand, the correct handling of the concepts of terminability and palliation could make a difference from the beginning of the treatment: it contributes to the correct information provided to the relatives or caretakers after the diagnosis, the correct management of stages of the disease and the type of care to be performed, not to generate false expectations in patients and relatives, to reduce visits for medical emergencies, to anticipate the patient's possible symptoms and to determine the tools and medications to be used for monitoring.

In the advanced stage, cancer requires a home treatment that includes the provision of palliative care that involves the management and evaluation of symptoms, hygiene care and administration of medications..$^{20}$ In the present study, it was observed that what is sought is to decrease the symptoms that are in progression, especially the pain that is the main symptom indicated. However, the focus is not only on the physical aspect of the patient but also on the psychological aspect. It is necessary to consider that providing care to patients with terminal illnesses may negatively affect family members who are not well prepared. ${ }^{21}$ For this reason, it is emphasized the importance of horizontal and constant communication between health personnel and family members, for responsible management of palliative care in the patient; While more information was provided to the family caregiver, visits to the emergency unit are expected to be minor.

The use of ICT in the form of communication devices was widely demonstrated among health professionals and caregivers interviewed. The aforementioned benefits, such as ease of communication with patients and distance monitoring of their status, are in line with those described by a qualitative study carried out in Scotland, which evaluated both the patients 'and professionals' perceptions with respect to the use of a distance self-monitoring system through mobile devices. ${ }^{22}$ where frequent face-to-face contact with health professionals may be difficult, the ongoing review and management of symptoms--a fundamental part of good palliative care--can be difficult to achieve. Telecare and other developments in information technology are increasingly being sought as a means of addressing shifting population demographics and rising demands on stretched health services, and may help in providing a system which allows patients to report their symptoms as they are happening. This may 
be one way of enhancing symptom management and improving quality of care at the end-of-life. A study testing the feasibility of using mobile phone-based technology Advanced Symptom Management System in Palliative Care (ASyMSp). However, in the case of patients and their family environment, a smaller use of ICTs was observed with respect to the mentioned study. This could be due to the precarious socioeconomic condition and the smaller access to the internet in general that the majority of patients visited had. Despite this, the TeleJampiq project will not limit its implementation in other types of patients. On contrary, the target population of the project will be composed of patients from different socioeconomic strata.

Another point of consensus among health professionals and caregivers was the utility that an electronic system would have for the recording of patient information, real-time monitoring, among others. A review of telehealth systems in palliative care that has been implemented in the United Kingdom (UK) describes the advantages of complementing these systems with a digital record of information, among which we have: faster access for health professionals, better use of time and improvement in the quality of the service provided.${ }^{17}$ With respect to the multidisciplinary approach, mentioned by health professionals as a vital point in the quality care of palliative and chronic patients, the literature shows a clear effectiveness of this type of equipment to achieve better management of these patients. ${ }^{23}$

Some of the emerging themes identified during the interviews were pain management, non-systematic types of knowledge, unnecessary monitoring in terminally ill patients, and multidisciplinary dimension for palliative care. These fields have also been shown to be potentially improved by the use of ICTs; however, the analysis of the exact form of implementation will require further studies in the field. ${ }^{24,25}$

There were many limitations to the present study. First, a convenience sample was used to select respondents. Although, we include health professionals from a public and a private institution, reflecting different care realities, this was not representative of the full range of chronic and palliative care offered in Peru. Second, ICTs which were mostly used by health professionals, family members or caregivers included cellphones, which limited the analysis of information obtained on more complex ICTs such as information systems or telemedicine systems. Finally, for reasons of accessibility, it was not decided to directly interview the patients; however, it would have been helpful for the study to be able to collect its own points of view.

\section{CONCLUSIONS}

One of the great challenges for this millennium, in addition to preventing chronic diseases and cancer, is how to deal with the management of chronic aspects and palliative care, looking for efficiency, effectiveness, comfort and the human side of improving the QoL of the patient. There is great potential in the field of telemedicine and palliative care that should be explored in order to reduce costs for both the patient and health system, and to prevent complications and improve patients' QoL, their caregivers and families. In this sense, and as seen throughout all the interviews, the use of ICTs in the field of chronic and palliative care has benefits not only in patient-physician communication, but also for training and reinforcing attitudes to family members and caregivers, for the adequate management of information and to improve the interdisciplinary care of patients.

\section{ACKNOWLEDGMENTS}

This article was supported by the National Program of Innovation for Competitiveness and Productivity (Innovate Peru) of the Peruvian Ministry of Production through the following contract $\mathrm{N}^{\circ}$ 203-PNICP-PIAP-2015. Its contents are solely the responsibility of the authors and do not represent the official views of the Peruvian Ministry of Production. The funders had no role in study design, data collection and analysis, decision to publish, or preparation of the manuscript.

\section{CONFLICTS OF INTEREST}

The authors declare that they have no conflicts of interest.

\section{REFERENCES}

1. Frenk J. Globalization and health: The role of knowledge in an interdependent world. Natl Institutes Heal. 2009; 1-10. Web site. http://www.fic.nih.gov/News/Events/Documents/barmes2009-transcript-julio-frenk.pdf. Accessed February 28, 2017.

2. Chan M. Climate change and health: Preparing for unprecedented challenges. The 2007 David E. Barmes Global Health Lecture. 2007. Web site. http://www.who.int/dg/speeches/2007/20071211_maryland/en/. Accessed January 11, 2017.

3. Bosch X. Two billion people older than 60 years by 2050 warns UN Secretary General. Lancet. 2002; 359(9314): 1321.

4. Sepúlveda C, Marlin A, Yoshida T, Ullrich A. Palliative Care: The World Health Organization's global perspective. J Pain Symptom Manage. 2002; 24(2): 91-96. doi: 10.1016/S08853924(02)00440-2

5. Duursma F, Schers HJ, Vissers KC, Hasselaar J. Study protocol: Optimization of complex palliative care at home via telemedicine. A cluster randomized controlled trial. BMC Palliat Care. 2011; 10: 13. doi: 10.1186/1472-684X-10-13

6. Van Den Berg N, Schumann M, Kraft K, Hoffmann W. Telemedicine and telecare for older patients-A systematic review. Maturitas. 2012; 73(2): 94-114. doi: 10.1016/j.maturitas.2012.06.010

7. Yang HL, Hsiao SL. Mechanisms of developing innovative IT-enabled services: A case study of Taiwanese healthcare 
service. Technovation. 2009; 29(5): 327-337. doi: 10.1016/j. technovation.2009.01.006

8. Capurro D, Ganzinger M, Pérez-Lu JE. Palliative care from a medical informatics perspective in Chile, Germany, and Peru. Stud Health Technol Inform. 2013; 192(1-2): 1013. doi: 10.3233/978-1-61499-289-9-1013

9. Ricur G, Carnicero J, Fernández A. Telemedicina: Generalidades y áreas de aplicación clínica. In: Manual de Salud Electrónica Para Directivos de Servicios Y Sistemas de Salud [In spanish]. México D.F., México: Naciones Unidas; 2011: 169193.

10. Chaudhry B, Wang J, Wu S, et al. Systematic review : Impact of health information technology on quality, efficiency, and costs of medical care. Ann Intern Med. 2006; 144: 742-752. doi: 10.7326/0003-4819-144-10-200605160-00125

11. Van Der Meijden MJ, Tange HJ, Troost J, Hasman A. Determinants of success of inpatient clinical information systems: A literature review. J Am Med Inform Assoc. 2003; 10(3): 235-243. doi: 10.1197/jamia.M1094

12. Mohd H, Mastura S, Mohamad S. Acceptance model of electronic medical record. J Adv Inf Manag Stud. 2005; 2(1): 75-92. Web site. http://repo.uum.edu.my/2246/. Accessed February 28, 2017.

13. Lober WB, Quiles C, Wagner S, et al. Three years experience with the implementation of a networked electronic medical record in Haiti. AMIA Annu Symp Proc. 2008: 434-438. Web site. https://pdfs.semanticscholar.org/04e9/baad513ab99fe68a87a271afae4110e14c4e.pdf. Accessed February 28, 2017.

14. Hersh WR. Adding value to the electronic health record through secondary use of data for quality assurance, research, and surveillance. Am J Manag Care. 2007; 13(6 Part 1): 277278. doi: 10.1001/jama.229.4.457

15. About Telemedicine. American Telemedicine Association. 2016. Web site. http://www.americantelemed.org/about/abouttelemedicine. Accessed February 28, 2017.

16. Ekeland AG, Bowes A, Flottorp S. Methodologies for assessing telemedicine: A systematic review of reviews. Int J Med
Inform. 2012; 81(1): 1-11. doi: 10.1016/j.ijmedinf.2011.10.009

17. Kidd L, Cayless S, Johnston B, Wengstrom Y. Telehealth in palliative care in the UK: A review of the evidence. $J$ Telemed Telecare. 2010; 16(7): 394-402. doi: 10.1258/jtt.2010.091108

18. Capurro D, Ganzinger M, Perez-Lu J, Knaup P. Effectiveness of ehealth interventions and information needs in palliative care: A systematic literature review. J Med Internet Res. 2014; 16(3): e72. doi: 10.2196/jmir.2812

19. Reigada C, Pais-ribeiro J. The caregiver role in palliative care: A systematic review of the literature. Heal Care Curr Rev. 2015; 3(2): 8-10. doi: 10.4172/2375-4273.1000143

20. Wittenberg-Lyles E, Parker Oliver D, Demiris G, Swarz J, Rendo M. YouTube as a tool for pain management with informal caregivers of cancer patients: A systematic review. J Pain Symptom Manage. 2014; 48(6): 1200-1210. doi: 10.1016/j.jpainsymman.2014.02.015

21. Brandstätter M, Kögler M, Baumann U, et al. Experience of meaning in life in bereaved informal caregivers of palliative care patients. Support Care Cancer. 2014; 22(5): 1391-1399. doi: 10.1007/s00520-013-2099-6

22. McCall K, Keen J, Farrer K, et al. Perceptions of the use of a remote monitoring system in patients receiving palliative care at home. Int J Palliat Nurs. 2008; 14(9): 426-431. doi: 10.12968/ ijpn.2008.14.9.31121

23. Oishi A, Murtagh FEM. The challenges of uncertainty and interprofessional collaboration in palliative care for non-cancer patients in the community: A systematic review of views from patients, carers and health-care professionals. Palliat Med. 2014; 28(9): 1081-1098. doi: 10.1177/0269216314531999

24. Allsop MJ, Taylor S, Mulvey MR, Bennett MI, Bewick BM. Information and communication technology for managing pain in palliative care: A review of the literature. BMJ Support Palliat Care. 2015; 5(5): 481-489. doi: 10.1136/bmjspcare-2013-000625

25. Reis A, Pedrosa A, Dourado M, Reis C. Information and communication technologies in long-term and palliative care. Procedia Technol. 2013; 9: 1303-1312. doi: 10.1016/j.protcy. 2013.12.146 